This is a preprint of: "Maximum number of limit cycles for certain piecewise linear dynamical systems", Jaume Llibre, Douglas D. Novaes, Marco Antonio Teixeira, Nonlinear Dynam., vol. 82(3), 1159-1175, 2015.

DOI: $[10.1007 / \mathrm{s} 11071-015-2223-\mathrm{x}]$

\title{
MAXIMUM NUMBER OF LIMIT CYCLES FOR CERTAIN PIECEWISE LINEAR DYNAMICAL SYSTEMS
}

\author{
JAUME LLIBRE ${ }^{1}$, DOUGLAS D. NOVAES ${ }^{1,2}$ AND MARCO A. TEIXEIRA ${ }^{2}$
}

\begin{abstract}
In this paper we compute the maximum number of limit cycles of some classes of planar discontinuous piecewise linear differential systems defined in two half-planes separated by a straight line $\Sigma$. Here we only consider non-sliding limit cycles, i.e. the limit cycle does not contain any sliding segment. Among all the cases that we study, in particular, we prove that this maximum number of limit cycles is 2, 1 or 1 if one of the two linear differential systems of the discontinuous piecewise linear differential system is a focus in $\Sigma$, a center, or a weak saddle, respectively. We also provide some normal forms for these systems.
\end{abstract}

\section{INTRODUCTION AND STATEMENT OF THE MAIN RESUlts}

Nonsmooth dynamical system appear in a natural way modelling many real processes and phenomena, for instance, recently piecewise linear differential equations appeared as idealized models of cell activity, see [9, 32, 33]. Due to that, in these last years the mathematical community became very interested in understanding the dynamics of these kind of system. In general, some of the main source of motivation to study nonsmooth systems can be found in control theory [4], impact and friction mechanics [5, 7, 24], nonlinear oscillations [1, 30], economics [16, 21], and biology [3, 23]. See for more details the book [11] and the references therein. Particularly the study of continuous and discontinuous piecewise linear differential systems has started with Andronov and coworkers [1].

Lum and Chua [29] in 1990 conjectured that a continuous piecewise linear vector field in the plane with two zones separated by a straight line, which is the easiest example of this kind of system, has at most one limit cycle. This conjecture was proved by Freire et al [13] in 1998. We remark that even in this relatively easy case, only after a serious work it was possible to show the existence of at most one limit cycle.

In this paper we consider the problem of Lum and Chua extended to the class of discontinuous piecewise linear differential systems in the plane with two

2010 Mathematics Subject Classification. 34C05, 34C07, 37G15.

Key words and phrases. discontinuous differential system, limit cycle, piecewise linear differential system. 
zones separated by a straight line, which is very related to the Hilbert's 16th problem [20] for this kind of system. Of course, limit cycles of discontinuous piecewise linear differential systems with two zones separated by a straight line have been studied recently by several authors, see among others $[2,8,10,12$, $14,15,17,18,19,25,26,27,28]$. Nevertheless the problem of Lum and Chua remains open for this class of differential equations. In this work we give a partial solution for this problem.

The difficulty in solving the problem of Lum and Chua for the class of differential equation that we are interested is due for basically two reasons. First, even being easy the computations of the solutions in any linear region, the time that each orbit requires to pass from one linear region to the other is not easy to compute, and consequently the matching of the corresponding solutions is a difficult problem. Second, the number of parameters to consider in order to be sure that one controls all possible configurations is generally not small, so the obtention of efficient canonical forms with fewer parameters is important.

It is worth to say that when the whole family of planar piecewise linear differential systems with two zones separated by a continuous curve is considered, then the maximum number of limit cycles that a system of this family can have does not exist. It is a consequence of a conjecture stated by Braga and Mello in [6] and then proved by Novaes and Ponce in [31].

In this paper we deal with planar vector fields $Z$ expressed as $\dot{z}=F(z)+$ $\operatorname{sign}(x) G(z)$, where $z=(x, y) \in \mathbb{R}^{2}$, and $F$ and $G$ are linear vector fields in $\mathbb{R}^{2}$ or, equivalently,

$$
\dot{z}= \begin{cases}X(z) & \text { if } \quad x>0, \\ Y(z) & \text { if } \quad x<0,\end{cases}
$$

where $X(z)=(F(z)+G(z)) / 2$ and $Y(z)=(F(z)-G(z)) / 2$. The line $\Sigma=\{x=$ $0\}$ is called discontinuous set. Our main goal is to study the maximum number of limit cycles that the discontinuous piecewise linear differential system (1) can have.

The systems $\dot{z}=X(z)$ and $\dot{z}=Y(z)$ are called lateral linear differential systems (or just lateral systems) and more specifically right system and left system, respectively.

A linear system is degenerate if the determinant of system is zero, otherwise is non-degenerate. System (1) can be classified according to the singularities of the lateral linear differential systems.

A non-degenerate linear differential system can have the following singularities: saddle $(S)$, node $(N)$, focus $(F)$, and center $(C)$. Among the above classes of singularities we shall also distinguish the following ones: a weak 
saddle, i.e. a saddle such that the sum of its eigenvalues is zero $\left(S^{0}\right)$; a diagonalizable node with distinct eigenvalues $(D N)$; a diagonalizable node with equal eigenvalues or star node $(\overline{D N})$; and a non-diagonalizable node $(n D N)$. We say that the discontinuous differential system (1) is a $L R$-system with $L, R \in\left\{S, S^{0}, D N, \overline{D N}, n D N, F, C\right\}$, when the left system has a singularity of type $L$ and the right system has a singularity of type $R$.

We define subclasses of $L R$-systems according to the position of the singularity of each lateral system. The right system can have a virtual singularity $\left(R_{v}\right)$, i.e. a singularity $p=\left(p_{x}, p_{y}\right)$ with $p_{x}<0$; a tangency singularity $\left(R_{d}\right)$, i.e. a singularity $p=\left(p_{x}, p_{y}\right)$ with $p_{x}=0$; or a real singularity $\left(R_{r}\right)$ i.e. a singularity $p=\left(p_{x}, p_{y}\right)$ with $p_{x}>0$. The left system can have a virtual singularity $\left(L_{v}\right)$, i.e. a singularity $p=\left(p_{x}, p_{y}\right)$ with $p_{x}>0$; a tangency singularity $\left(L_{d}\right)$, i.e. a singularity $p=\left(p_{x}, p_{y}\right)$ with $p_{x}=0$; or a real singularity $\left(L_{r}\right)$ i.e. a singularity $p=\left(p_{x}, p_{y}\right)$ with $p_{x}<0$.

We denote by $\mathcal{N}(L, R)$ the maximum number of non-sliding limit cycles that a $L R$-system can have. Here a non-sliding limit cycles is a limit cycle that does not contain any sliding segment in $\Sigma$. Clearly $\mathcal{N}(L, R)=\mathcal{N}(R, L)$.

In this paper we prove that $\mathcal{N}(L, R) \leq 2$ always when one of the lateral systems is a degenerate linear differential system, a saddle of kind $S_{v}, S_{d}, S^{0}$, a node of kind $D N_{r}, D N_{d}, \overline{D N}, n D N_{r}, n D N_{d}$, a focus of kind $F_{d}$, and a $C$. Moreover we compute the exact value of $\mathcal{N}(L, R)$ in all the above cases.

If one of the lateral systems is a degenerate linear differential system, then system (1) does not admits the existence of limit cycles. Indeed, for a degenerate linear differential system always there exists a straight line of singularities such that the solutions are all either parallel or perpendicular to this line. So the first return map on the straight line $x=0$ is not defined. It means that $\mathcal{N}(L, R)=0$ in this case.

It is easy to see that if one of the lateral linear differential systems is of type $S_{v}, S_{d}, D N_{r}, D N_{d}, \overline{D N}, n D N_{r}$, or $n D N_{d}$, then the first return map on the straight line $x=0$ of system (1) is not defined. Consequently system (1) does not admit limit cycles in all these cases. So $\mathcal{N}(R, L)=0$ for the systems having one of these kind of equilibria.

It remains to study the cases when one of the lateral system is $F_{d}, C$ or $S_{r}^{0}$. For studying such cases we shall use the following theorems.

Theorem 1. All numbers $\mathcal{N}\left(F_{d}, F_{v}\right), \mathcal{N}\left(F_{d}, F_{r}\right), \mathcal{N}\left(F_{d}, D N_{v}\right), \mathcal{N}\left(F_{d}, n D N_{v}\right)$ and $\mathcal{N}\left(F_{d}, S_{r}\right)$ are equal to 2 , and all numbers $\mathcal{N}\left(F_{d}, F_{d}\right), \mathcal{N}\left(F_{d}, C\right)$ and $\mathcal{N}\left(F_{d}, S_{r}^{0}\right)$ are equal to 1.

Theorem 2. All numbers $\mathcal{N}\left(S_{r}^{0}, F\right), \mathcal{N}\left(S_{r}^{0}, D N_{v}\right), \mathcal{N}\left(S_{r}^{0}, n D N_{v}\right)$ and $\mathcal{N}\left(S_{r}^{0}, S_{r}\right)$ are equal to 1 , and all numbers $\mathcal{N}\left(S_{r}^{0}, C\right)$ and $\mathcal{N}\left(S_{r}^{0}, S_{r}^{0}\right)$ are equal to 0. 
We shall see that the next result can be obtained as an immediately corollary of the proofs of Theorems 1 and 2 .

Corollary 3. All numbers $\mathcal{N}\left(C_{d}, F\right), \mathcal{N}\left(C_{d}, D N_{v}\right), \mathcal{N}\left(C_{d}, n D N_{v}\right)$ and $\mathcal{N}\left(C_{d}, S_{r}\right)$ are equal to 1 , and all numbers $\mathcal{N}\left(C_{d}, C\right)$ and $\mathcal{N}\left(C_{d}, S_{r}^{0}\right)$ are equal to 0.

The equalities of Corollary 3 can be extended for all linear centers.

Theorem 4. All numbers $\mathcal{N}(C, F), \mathcal{N}\left(C, D N_{v}\right), \mathcal{N}\left(C, n D N_{v}\right)$ and $\mathcal{N}\left(C, S_{r}\right)$ are equal to 1 , and all numbers $\mathcal{N}(C, C)$ and $\mathcal{N}\left(C, S_{r}^{0}\right)$ are equal to 0.

\section{Preliminary Results}

A linear change of variables in the plane preserving the vertical lines will be called in what follows a vertical lines-preserving linear change of variables.

Proposition 5. Let $M=\left(m_{i j}\right)_{i, j}$ be a $2 \times 2$ matrix. If the linear differential system

$$
(\dot{x}, \dot{y})^{T}=M(x, y)^{T}
$$

is a

(a) S-system then after a vertical lines-preserving linear change of variables and a time-rescaling system (2) becomes $(\dot{x}, \dot{y})^{T}=M_{1}(x, y)^{T}$;

(b) DN-system then after a vertical lines-preserving linear change of variables and a time-rescaling system (2) becomes $(\dot{x}, \dot{y})^{T}=M_{2}(x, y)^{T}$;

(c) F-system then after a vertical lines-preserving linear change of variables and a time-rescaling system (2) becomes $(\dot{x}, \dot{y})^{T}=M_{3}(x, y)^{T}$;

(d) nDn-system then after a vertical lines-preserving linear change of variables and a time-rescaling system (2) becomes $(\dot{x}, \dot{y})^{T}=M_{4}(x, y)^{T}$, where

$$
\begin{array}{ll}
M_{1}=\left(\begin{array}{ll}
a & 1 \\
1 & a
\end{array}\right) \quad \text { with } \quad|a|<1 ; & M_{2}=\left(\begin{array}{cc}
a & 1 \\
1 & a
\end{array}\right) \quad \text { with } \quad|a|>1 ; \\
M_{3}=\left(\begin{array}{cc}
a & 1 \\
-1 & a
\end{array}\right) \quad \text { with } \quad a \in \mathbb{R} ; \quad \text { and } \quad M_{4}=\left(\begin{array}{cc}
\lambda & \lambda \\
0 & \lambda
\end{array}\right) \quad \text { with } \lambda= \pm 1 .
\end{array}
$$

Proof of Proposition 5. Let $S=\left(s_{i j}\right)_{i, j}$ be a $2 \times 2$ matrix. The change of variables $(u, v)^{T}=S(x, y)^{T}$ is a vertical lines-preserving linear change of variables if and only if $s_{12}=0$ and $s_{11}=1$. Indeed, $S(x, y)=\left(s_{11} x+s_{12} y, s_{21} x+s_{22} y\right)$ and $s_{11} x+s_{12} y=x$ for every $x \in \mathbb{R}$ if and only if $s_{11}=1$ and $s_{12}=0$. So in what follows we fix $s_{12}=0$ and $s_{11}=1$.

Claim 1. The statement (a) holds. 
Taking

$$
s_{21}=\frac{m_{11}-m_{22}}{\sqrt{4 m_{12} m_{21}+\left(m_{11}-m_{22}\right)^{2}}}, \quad \text { and } \quad s_{22}=\frac{2 m_{12}}{\sqrt{4 m_{12} m_{21}+\left(m_{11}-m_{22}\right)^{2}}},
$$

it follows that

$$
S M S^{-1}=\frac{1}{2}\left(\begin{array}{cc}
m_{11}+m_{22} & \sqrt{4 m_{12} m_{21}+\left(m_{11}-m_{22}\right)^{2}} \\
\sqrt{4 m_{12} m_{21}+\left(m_{11}-m_{22}\right)^{2}} & m_{11}+m_{22}
\end{array}\right) .
$$

We note that $\sqrt{4 m_{12} m_{21}+\left(m_{11}-m_{22}\right)^{2}} \neq 0$, because otherwise the singularity would be a node with equal eigenvalues $\overline{D N}$. Then we can rescale the time by

$$
\tau=\sqrt{4 m_{12} m_{21}+\left(m_{11}-m_{22}\right)^{2}} t .
$$

Denoting $a=\left(m_{11}+m_{22}\right) / \sqrt{4 m_{12} m_{21}+\left(m_{11}-m_{22}\right)^{2}}$ system (2) becomes

$$
\left(\begin{array}{l}
x^{\prime} \\
y^{\prime}
\end{array}\right)=\left(\begin{array}{ll}
a & 1 \\
1 & a
\end{array}\right)\left(\begin{array}{l}
x \\
y
\end{array}\right) \text {. }
$$

where now the prime denotes the derivative with respect to the new time variable $\tau$. Computing the eigenvalues of the above system $\{-1+a, 1+a\}$ we conclude that $|a|<1$, because this system is a saddle, i.e. the eigenvalues have different sign. Therefore we have proved statement $(a)$.

Claim 2. The statement (b) holds.

The proof of statement $(b)$ follows similar to the proof of statement $(a)$. Nevertheless we conclude that $|a|>1$, because in this case the system is a diagonalizable node, i.e. the eigenvalue have the same sign. Thus we have proved statement $(b)$.

Claim 3. The statement (c) holds.

Taking

$$
s_{21}=\frac{m_{11}-m_{22}}{\sqrt{-4 m_{12} m_{21}-\left(m_{11}-m_{22}\right)^{2}}}, \quad \text { and } \quad s_{22}=\frac{2 m_{12}}{\sqrt{-4 m_{12} m_{21}-\left(m_{11}-m_{22}\right)^{2}}},
$$

it follows that

$$
S M S^{-1}=\frac{1}{2}\left(\begin{array}{cc}
m_{11}+m_{22} & \sqrt{-4 m_{12} m_{21}-\left(m_{11}-m_{22}\right)^{2}} \\
-\sqrt{-4 m_{12} m_{21}-\left(m_{11}-m_{22}\right)^{2}} & m_{11}+m_{22}
\end{array}\right) .
$$


From hypotheses this system is a focus thus $\sqrt{-4 m_{12} m_{21}-\left(m_{11}-m_{22}\right)^{2}}>0$. So we can rescale the time by $\tau=\sqrt{-4 m_{12} m_{21}-\left(m_{11}-m_{22}\right)^{2}} t$. Denoting $a=\left(m_{11}+m_{22}\right) / \sqrt{4 m_{12} m_{21}+\left(m_{11}-m_{22}\right)^{2}}$ system (2) becomes

$$
\left(\begin{array}{l}
x^{\prime} \\
y^{\prime}
\end{array}\right)=\left(\begin{array}{cc}
a & 1 \\
-1 & a
\end{array}\right)\left(\begin{array}{l}
x \\
y
\end{array}\right) .
$$

where now the prime denotes the derivative with respect to the new time variable $\tau$. Computing the eigenvalues of the above system $\{-i+a, i+a\}$ we conclude that $a \neq 1$, because this system is a focus. Hence statement $(c)$ is proved.

Claim 4. The statement $(d)$ holds.

One of the entries $m_{12}$ or $m_{21}$ are distinct of zero. Indeed, Suppose that $m_{12}=0$, so $\left\{m_{11}, m_{22}\right\}$ are the eigenvalues of the matrix $M$. Since system (2) is a non-diagonalizable node we have that $m_{11}=m_{22}$ which implies that $m_{21} \neq 0$, in other way the matrix $M$ would be diagonalizable. On the other hand, supposing that $m_{21}=0$ we obtain $m_{12} \neq 0$. From here we assume without loss of generality that $m_{12} \neq 0$.

We also have that $m_{11}+m_{22} \neq 0$. Indeed, in this case $\pm \sqrt{m_{11}^{2}+m_{12} m_{21}}$ are the eigenvalues of the matrix $M$. Again, since system (2) is a nondiagonalizable node we have that the matrix $M$ has only one eigenvalue with multiplicity 2. This implies that $\sqrt{m_{11}^{2}+m_{12} m_{21}}=0$, which is a contradiction. It is also easy to see that $4 m_{12} m_{21}+\left(m_{11}-m_{22}\right)^{2}=0$.

Taking

$$
s_{21}=\frac{m_{11}-m_{22}}{2 m_{12}} \quad \text { and } \quad s_{22}=\frac{2 m_{12}}{m_{11}+m_{22}},
$$

it follows that

$$
S M S^{-1}=\frac{1}{2}\left(\begin{array}{cc}
m_{11}+m_{22} & m_{11}+m_{22} \\
0 & m_{11}+m_{22}
\end{array}\right) .
$$

So we can rescale the time by $\tau=\left|m_{11}+m_{22}\right| t$ system (2) becomes

$$
\left(\begin{array}{l}
x^{\prime} \\
y^{\prime}
\end{array}\right)=\left(\begin{array}{ll}
\lambda & \lambda \\
0 & \lambda
\end{array}\right)\left(\begin{array}{l}
x \\
y
\end{array}\right) \text {. }
$$

where $\lambda= \pm 1$, and now the prime denotes the derivative with respect to the new time variable $\tau$. This completes the proof of statement $(c)$. 
We say that an ordered set of complex-valued functions $F=\left(f_{0}, f_{1}, \ldots, f_{n}\right)$ defined on $I$ is an Extended Chebyshev system or ET-system on $I$ if and only if any nontrivial linear combination of the functions of $F$ has at most $n$ zeros counting multiplicities. We say that $F$ is an Extended Complete Chebyshev system or an ECT-system on $I$ if and only if for any $k, 0 \leq k \leq n,\left(f_{0}, f_{1}, \ldots, f_{k}\right)$ is an ET-system. For more details, see the book of Karlin and Studden [22].

In order to prove that $F$ is a ECT-system on $I$ it is sufficient and necessary to show that $W\left(f_{0}, f_{1}, \ldots, f_{k}\right)(t) \neq 0$ on $I$ for $0 \leq K \leq n$. Here $W\left(f_{0}, f_{1}, \ldots, f_{k}\right)(t)$ denotes the Wronskians of the functions $\left(f_{0}, f_{1}, \ldots, f_{k}\right)$ with respect to $t$. We recall the definition of the Wronskian.

$$
W\left(f_{0}, f_{1}, \ldots, f_{k}\right)(t)=\left|\begin{array}{cccc}
f_{0}(t) & f_{1}(t) & \cdots & f_{k}(t) \\
f_{0}^{\prime}(t) & f_{1}^{\prime}(t) & \cdots & f_{k}^{\prime}(t) \\
\vdots & \vdots & \ddots & \vdots \\
f_{0}^{(k)}(t) & f_{1}^{(k)}(t) & \cdots & f_{k}^{(k)}(t)
\end{array}\right|
$$

Now consider the functions

$$
\begin{array}{ll}
\xi_{1}(t)=1, & \\
\xi_{2}^{1}(t)=\cot (t)-e^{a t} \csc (t), & \xi_{2}^{2}(t)=\operatorname{coth}(t)-e^{a t} \operatorname{csch}(t), \\
\xi_{3}^{1}(t)=\cot (t)-e^{-a t} \csc (t), & \xi_{2}^{3}(t)=\operatorname{coth}(t)-e^{-a t} \operatorname{csch}(t), \\
\xi_{3}^{2}=\frac{1-e^{t}}{t}, & \xi_{3}^{3}=\frac{1-e^{-t}}{t}, \\
\xi_{2}^{4}(t)=\csc (t) \sinh (a t), & \xi_{2}^{5}(t)=\operatorname{csch}(t) \sinh (a t), \\
\xi_{2}^{6}(t)=\frac{\sinh (t)}{t} . &
\end{array}
$$

We define the sets of functions $\mathcal{F}^{i}=\left\{\xi_{1}, \xi_{2}^{i}, \xi_{3}^{i}\right\}$ for $i=1,2,3$, and $\mathcal{F}^{i}=$ $\left\{\xi_{1}, \xi_{2}^{i}\right\}$ for $i=4,5,6$.

Lemma 6. The following statements hold.

(a) The set of functions $\mathcal{F}^{1}$ is a ECT-system on the intervals $(0, \pi)$ and $(-\pi, 0)$ for every $a \neq 0$.

(b) The set of functions $\mathcal{F}^{2}$ is a ECT-system on $\mathbb{R}^{+}$for every $a \notin\{0, \pm 1\}$.

(c) The set of functions $\mathcal{F}^{3}$ is a ECT-system on $\mathbb{R}^{+}$.

(d) The set of functions $\mathcal{F}^{4}$ is a ECT-system on the intervals $(0, \pi)$ and $(-\pi, 0)$ for every $a \neq 0$.

(e) The set of functions $\mathcal{F}^{5}$ is a ECT-system on $\mathbb{R}^{+}$for every a $\notin\{0, \pm 1\}$.

(f) The set of functions $\mathcal{F}^{6}$ is a ECT-system on $\mathbb{R}^{+}$. 
Proof. To prove the statements $(a)-(f)$ we compute the Wronskians $W_{1}(t)=$ $W\left(\xi_{1}\right)(t), W_{2}^{i}(t)=W\left(\xi_{1}, \xi_{2}^{i}\right)(t)$ for $i=1,2, \ldots, 6$, and $W_{3}^{i}(t)=W\left(\xi_{1}, \xi_{2}^{i}, \xi_{3}^{i}\right)(t)$ for $i=1,2,3$.

$$
\begin{aligned}
& W_{1}(t)=1, \\
& W_{2}^{1}(t)=\csc (t)\left(e^{a t}(\cot (t)-a)-\csc (t)\right), \\
& W_{3}^{1}(t)=2\left(1+a^{2}\right) \csc ^{2}(t)(\csc (t) \sinh (a t)-a), \\
& W_{2}^{2}(t)=\operatorname{csch}(t)\left(e^{a t}(\operatorname{coth}(t)-a)-\operatorname{csch}(t)\right), \\
& W_{3}^{2}(t)=2\left(1-a^{2}\right) \operatorname{csch}^{2}(t)(\operatorname{csch}(t) \sinh (a t)-a), \\
& W_{2}^{3}=\frac{e^{t}(1-t)-1}{t^{2}}, \\
& W_{3}^{3}=\frac{2(t-\sinh (t))}{t^{3}}, \\
& W_{2}^{4}(t)=\csc (t)(a \cosh (a t)-\cot (t) \sinh (a t)), \\
& W_{2}^{5}(t)=\operatorname{csch}(t)(a \cosh (a t)-\operatorname{coth}(t) \sinh (a t)), \\
& W_{2}^{6}(t)=\frac{t \cosh (t)-\sinh (t)}{t^{2}}
\end{aligned}
$$

From here it is easy to see that for each $a \neq 0$ the Wronskians $W_{2}^{1}, W_{3}^{1}$ and $W_{2}^{4}$ do not vanish at any point of the interval $(0, \pi)$ or $(-\pi, 0)$; for each $a \notin\{0, \pm 1\}$ the Wronskians $W_{2}^{2}, W_{3}^{2}$ and $W_{2}^{5}$ do not vanish at any point of $\mathbb{R}^{+}$; and the Wronskians $W_{2}^{3}, W_{3}^{3}$ and $W_{2}^{6}$ do not vanish at any point of $\mathbb{R}^{+}$. So the lemma is proved.

For $a=0$ we define the sets of functions $\mathcal{G}^{i}=\left\{\xi_{1}, \xi_{2}^{i}\right\}$ for $i=1,2$. Lemma 6 is stated assuming $a \neq 0$. When $a=0$ we will need the following lemma.

Lemma 7. Assume that $a=0$. Then following statements hold.

(a) The set of functions $\mathcal{G}^{1}$ is a ECT-system on the intervals $(0, \pi)$ and $(-\pi, 0)$.

(b) The set of functions $\mathcal{G}^{2}$ is a ECT-system on $\mathbb{R}^{+}$. 
Proof. Proceeding analogously to the proof of Lemma 6 we compute the Wronskians.

$$
\begin{aligned}
& W_{1}(t)=1, \\
& W_{2}^{1}(t)=\csc (t) \cot (t)-\csc ^{2}(t), \\
& W_{2}^{2}(t)=\operatorname{csch}(t) \operatorname{coth}(t)-\operatorname{csch}^{2}(t) .
\end{aligned}
$$

From here it is easy to see that the Wronskian $W_{2}^{1}$ does not vanish at any point of the interval $(0, \pi)$ or $(-\pi, 0)$, and that the Wronskian $W_{2}^{2}$ does not vanish at any point of $\mathbb{R}^{+}$.

Lemma 8. For $u_{1}>0$ we consider the functions

$$
\begin{aligned}
& F(t)=u_{1}\left(e^{-a t} \csc (t)-\cot (t)\right), \quad G(t)=u_{1}\left(e^{-a t} \operatorname{csch}(t)-\operatorname{coth}(t)\right), \\
& H(t)=\frac{u_{1}\left(e^{-t}-1\right)}{t} .
\end{aligned}
$$

The following statements hold.

(a) For every $a \in \mathbb{R}, F(t)$ is a monotonic increasing function in the interval $(-\pi, \pi)$ such that $F(t)<-a u_{1}$ for $t \in(-\pi, 0)$, and $F(t)>-a u_{1}$ for $t \in(0, \pi)$.

(b) For $|a|>1 G(t)$ is a monotonic increasing function in $\mathbb{R}$ such that $G(t)>-a u_{1}$ for $t>0$; and for $|a|<1 G(t)$ is a monotonic decreasing function in $\mathbb{R}$ such that $G(t)>-a u_{1}$ for $t>0$.

(c) $H(t)$ is a monotonic increasing function such that $H(t)<-u_{1}$ for $t<0$, and $H(t)>-u_{1}$ for $t>0$.

Proof. To prove statement (a) we compute

$$
F^{\prime}(t)=u_{1} \csc ^{2}(t)\left(1-e^{-a t}(\cos (t)+a \sin (t))\right)=u_{1} \csc ^{2}(t) p(t),
$$

where $p(t)=1-e^{-a t}(\cos (t)+a \sin (t))$, and $p^{\prime}(t)=\left(1+a^{2}\right) e^{-a t} \sin (t)$. Now, if $u_{1}>0$ and $0<t<\pi$, then $p^{\prime}(t)>0$. Since $p(0)=0$ we conclude that $p(t)>0$ and $F^{\prime}(t)>0$ for every $0<t<\pi$, which implies that $F(t)>-a u_{1}$ for every $0<t<\pi$, because $\lim _{t \rightarrow 0} F(t)=-a u_{1}$. On the other hand, if $u_{1}<0$ and $-\pi<t<0$, then $p^{\prime}(t)<0$. So analogously we conclude that $F(t)>-a u_{1}$ for every $0<t<\pi$. Moreover, we have shown that the function $F$ is increasing for $0<t<\pi$ when $u_{1}>0$, and for $-\pi<t<0$ when $u_{1}<0$. 
To prove statement $(b)$ we compute

$$
\begin{aligned}
G^{\prime}(t) & =u_{1} \operatorname{csch}(t)\left(\operatorname{csch}(t)-e^{-a t}(a+\operatorname{coth}(t))\right) \\
& =\frac{e^{-a t} u_{1} \operatorname{csch}(t)}{\left(e^{t}-1\right)\left(e^{t}+1\right)}\left(a-1-e^{2 t}-a e^{2 t}+2 e^{t+a t}\right) \\
& =\frac{e^{-a t} u_{1} \operatorname{csch}(t)}{\left(e^{t}-1\right)\left(e^{t}+1\right)} q(t),
\end{aligned}
$$

where $q(t)=a-1-e^{2 t}-a e^{2 t}+2 e^{t+a t}$. We compute $q^{\prime}(t)=-2(1+a) e^{t}\left(e^{t}-e^{a t}\right)$. Now, if $|a|>1$, then $p^{\prime}(t)>0$ for every $t>0$. Since $q(0)=0$ we conclude that $q(t)>0$ and $G^{\prime}(t)>0$ for every $t>0$, which implies that $G(t)>-a u_{1}$ for every $t>0$, because $\lim _{t \rightarrow 0} G(t)=-a u_{1}$. On the other hand, if $|a|<1$, then $q^{\prime}(t)<0$ for every $t>0$. So analogously we conclude that $G(t)<-a u_{1}$ for every $t>0$. Moreover, we have shown that the function $G$ is increasing for $t>0$ when $|a|>1$, and that $G$ is decreasing for $t>0$ when $|a|<1$.

To prove statement $(c)$ we compute

$$
H^{\prime}(t)=\frac{u_{1} e^{-t}}{t^{2}}\left(e^{t}-t-1\right)=\frac{u_{1} e^{-t}}{t^{2}} r(t),
$$

where $r(t)=e^{t}-t-1$. We compute $r^{\prime}(t)=e^{t}-1>0$. Since $r(0)=0$ we conclude that $r(t)>0$ and $H^{\prime}(t)>0$ for every $t>0$, and that that $r(t)<0$ and $H^{\prime}(t)<0$ for every $t>0$. Thus we obtain that $H(t)>-u_{1}$ for every $t>0$, and that $H(t)<-u_{1}$ for every $t<0$, because $\lim _{t \rightarrow 0} H(t)=-u_{1}$. Moreover, we have shown that the function $H$ is increasing for $t \in \mathbb{R}$.

\section{Proof of Theorems 1, 2 and 4, and Corollary 3}

The proofs of Theorem 1 and Corollary 3 will be immediately consequences of Propositions 9 -14; the proof of Theorem 2 will be an immediately consequence of Propositions 14-19; and the proof of Theorem 4 will be an immediately consequence of Propositions 20-23 and Corollary 3.

We write system (1) as

$$
\left(\begin{array}{l}
\dot{x} \\
\dot{y}
\end{array}\right)=\left\{\begin{array}{l}
\left(\begin{array}{ll}
a_{11} & a_{12} \\
a_{21} & a_{22}
\end{array}\right)\left(\begin{array}{l}
x+u_{1} \\
y+u_{2}
\end{array}\right) \quad \text { if } \quad x>0, \\
\left(\begin{array}{ll}
b_{11} & b_{12} \\
b_{21} & b_{22}
\end{array}\right)\left(\begin{array}{l}
x+v_{1} \\
y+v_{2}
\end{array}\right) \quad \text { if } \quad x<0
\end{array}\right.
$$

The solution of the non-smooth piecewise linear differential system (4) can be easily computed, because it is a piecewise linear differential systems. So let $\varphi^{+}(t, x, y)=\left(\varphi_{1}^{+}(t, x, y), \varphi_{2}^{+}(t, x, y)\right)$ be the solution of (4) for $x>0$ such that 
$\varphi^{+}(0, x, y)=(x, y)$. Similarly, let $\varphi^{-}(t, x, y)=\left(\varphi_{1}^{-}(t, x, y), \varphi_{2}^{-}(t, x, y)\right)$ be the solution of (4) for $x<0$ such that $\varphi^{-}(0, x, y)=(x, y)$.

In what follows, let $t^{+}(y)$ be the smallest positive time such that $\varphi_{1}^{+}\left(t^{+}(y), 0\right.$, $y)=0$; let $t_{+}(y)$ be the biggest negative time such that $\varphi_{1}^{+}\left(t_{+}(y), 0, y\right)=0$; let $t^{-}(y)$ be the biggest negative time such that $\varphi_{1}^{-}\left(t^{-}(y), 0, y\right)=0 ; t_{-}(y)$ be the smallest positive time such that $\varphi_{1}^{-}\left(t_{-}(y), 0, y\right)=0$. Observe that the functions $t^{+}(y), t_{+}(y), t^{-}(y)$, and $t_{-}(y)$ are not necessarily always defined.

Assuming that $t^{+}(y)$ and $t^{-}(y)$ are defined then there exists a limit cycle passing through the point $(0, y)$ with $y \in J^{*}=\operatorname{Dom}\left(t^{+}\right) \cup \operatorname{Dom}\left(t^{-}\right)$if and only if $\varphi_{2}^{+}\left(t^{+}(y), 0, y\right)=\varphi_{2}^{-}\left(t^{-}(y), 0, y\right)$. Thus, in this case, we must study the zeros $y^{*}$ of the function

$$
f(y)=\varphi_{2}^{+}\left(t^{+}(y), 0, y\right)-\varphi_{2}^{-}\left(t^{-}(y), 0, y\right)
$$

on the domain $J^{*}$.

Of course if $t_{+}(y)$ and $t_{-}(y)$ are defined then there exists a limit cycle passing through $(0, y)$ with $y \in J_{*}=\operatorname{Dom}\left(t_{+}\right) \cup \operatorname{Dom}\left(t_{-}\right)$if and only if $\varphi_{2}^{+}\left(t_{+}(y), 0, y\right)=$ $\varphi_{2}^{-}\left(t_{-}(y), 0, y\right)$. Thus, in this case, we must study the zeros $y_{*}$ of the function

$$
f(y)=\varphi_{2}^{+}\left(t_{+}(y), 0, y\right)-\varphi_{2}^{-}\left(t_{-}(y), 0, y\right)
$$

on the domain $J_{*}$.

Since the vectors fields $X$ and $Y$ are linear, then a limit cycle passing through a point $\left(x_{0}, y_{0}\right)$ must contain points $\left(0, y^{*}\right)$ and $\left(0, y_{*}\right)$ such that $y^{*} \in J^{*}$ and $y_{*} \in J_{*}$.

Let $X=\left(X_{1}, X_{2}\right)$ and $Y=\left(Y_{1}, Y_{2}\right)$. We say that a point $(0, y)$ is an

(a) invisible fold point for the right system when

$$
X_{1}(0, y)=0 \quad \text { and } \quad \frac{X_{1}}{\partial y}(0, y) X_{2}(0,0)<0 ;
$$

(c) invisible fold point for the left system when

$$
Y_{1}(0, y)=0 \quad \text { and } \quad \frac{Y_{1}}{\partial y}(0, y) Y_{2}(0,0)>0 .
$$

An affine (linear) change of variables in the plane preserving the straight line $x=0$ will be called in what follows a $\Sigma$-preserving affine (linear) change of variables, and a $\Sigma$-preserving affine (linear) change of variables which also preserves the semiplane $x>0$ will be called in what follows a $\Sigma^{+}$-preserving affine (linear) change of variables. Clearly a $\Sigma^{+}$-preserving affine (linear) change of variables also preserves the semiplane $x<0$.

The case when the left system has a focus or a center on $\Sigma$ will be study in subsection 3.1, the case when the left system has a weak saddle will be study 
in subsection 3.2, and the case when the left system has a virtual or real center will be study in subsection 3.3.

3.1. Left system has a focus or a center on $\Sigma$. In this case $v_{1}=0$, $4 b_{12} b_{21}+\left(b_{11}-b_{22}\right)^{2}<0$ and the point $\left(0,-v_{2}\right)$ is a singularity of focus type.

Let $\Gamma=\sqrt{-4 b_{12} b_{21}-\left(b_{11}-b_{22}\right)^{2}}$. The function $t^{-}(y)$ is defined for every $y>-v_{2}$, and we compute $t^{-}(y)=-2 \pi / \Gamma$. Analogously the function $t_{-}(y)$ is defined for every $y<-v_{2}$, and we compute $t_{-}(y)=2 \pi / \Gamma$.

In order to fix the clockwise orientation of the flow of system (4) we assume that $Y_{1}\left(0,1-v_{2}\right)=b_{12}>0$.

Proposition 9. The equalities $\mathcal{N}\left(F_{d}, F_{v}\right)=2, \mathcal{N}\left(F_{d}, C_{v}\right)=\mathcal{N}\left(C_{d}, F_{v}\right)=1$ and $\mathcal{N}\left(C_{d}, C_{v}\right)=0$ hold.

Proof. From Proposition $5(c)$ we can assume that $a_{11}=a_{22}=a$ with $a \in \mathbb{R}$, $a_{12}=-a_{21}=1$, and by a $\Sigma^{+}$-preserving translation we can take $u_{2}=0$. Moreover $u_{1}>0$ because the right system has a focus which is virtual for system 4 .

It is easy to see that the point $\left(0,-a u_{1}\right) \in \Sigma$ is an invisible fold point for the right system. So the function $t^{+}(y)$ is defined for every $y>-a u_{1}$. On the other hand its image is the interval $(0, \pi)$. Indeed, given $y>-a u_{1}$ consider the line $\ell(y)$ passing through the focus point $\left(-u_{1}, 0\right)$ and $(0, y)$. The trajectory of the left system starting at $(0, y)$ returns to the line $\ell(y)$ at $t=\pi$, so it must return to $\Sigma$ in a time $t<\pi$. Thus $t^{+}(y) \in(0, \pi)$ for every $y>-a u_{1}$.

We know that $\varphi_{1}^{+}\left(t^{+}(y), 0, y\right)=0$ for every $y>-a u_{1}$, that is

$$
-u_{1}+e^{a t^{+}(y)}\left(u_{1} \cos \left(t^{+}(y)\right)+y \sin \left(t^{+}(y)\right)\right)=0 .
$$

Hence taking $y^{+}=\left.F\right|_{(0, \pi)}$ we have that $y^{+}\left(t^{+}(y)\right)=y$ for every $y>-a u_{1}$. The function $F$ is defined in (3).

Now we claim that $t^{+}\left(y^{+}(t)\right)=t$ for every $t \in(0, \pi)$. Indeed, for $t_{0} \in(0, \pi)$ let $y_{0}=y^{+}\left(t_{0}\right)$. From Lemma $8(a) y_{0}>-a u_{1}$, so from the above comments we obtain that $y_{0}=y^{+}\left(t^{+}\left(y_{0}\right)\right)$. Thus $y^{+}\left(t_{0}\right)=y^{+}\left(t^{+}\left(y_{0}\right)\right)$. Again from Lemma $8(a) y^{+}(t)=F(t)$ is injective on the interval $(0, \pi)$, so $t_{0}=t^{+}\left(y_{0}\right)$. Hence $t_{0}=t^{+}\left(y_{0}\right)=t^{+}\left(y^{+}\left(t_{0}\right)\right)$. Since $t_{0}$ was arbitrarily chosen in $(0, \pi)$ we conclude that $t^{+}\left(y^{+}(t)\right)=t$ for every $t \in(0, \pi)$. Therefore the function $t^{+}:\left(-a u_{1}, \infty\right) \rightarrow(0, \pi)$ is invertible with inverse equal to $y^{+}:(0, \pi) \rightarrow$ $\left(-a u_{1}, \infty\right)$.

Let $Y_{M}=\max \left\{-a u_{1},-v_{2}\right\}$, so computing the zeros of the function (5) for $y>Y_{M}$ is equivalent to compute the zeros of the function

$$
g_{1}(t)=f\left(y^{+}(t)\right)=k_{1} \xi_{1}+k_{2} \xi_{2}^{1}+k_{3} \xi_{3}^{1}
$$


for $t \in I \subset(0, \pi)$, where $k_{1}=v_{2}(1+\delta), k_{2}=u_{1}, k_{3}=-\delta u_{1}, \delta=e^{-\frac{\left(b_{11}+b_{22}\right) \pi}{\Gamma}}$, and $I=t^{+}\left(\left(Y_{M}, \infty\right)\right)$. Note that if $v_{2} \geq a u_{1}$ then $I=(0, \pi)$.

Applying Lemma $6(a)$ we conclude that $\mathcal{N}\left(F_{d}, F_{v}\right) \leq 2$. Now choosing $a=-1, u_{1}=8, v_{2}=8 / 9$, and $b_{i, j}$ for $i, j=1,2$ such that $\delta=1 / 8$ we obtain $k_{1}=-5, k_{2}=8$, and $k_{3}=-1$ which implies that $(7)$ has 2 zeros on $(0, \pi)$, namely $t_{1} \approx 0.770$ and $t_{2} \approx 2.203$. Since $y^{+}\left(t_{1}\right) \approx 16.572$ and $y^{+}\left(t_{2}\right) \approx 95.667$, for this choice of coefficients, there exists two limit cycles of system (4) passing respectively through the points $\left(0, y^{+}\left(t_{1}\right)\right)$ and $\left(0, y^{+}\left(t_{2}\right)\right)$.

Now, the right system is a center if and only if $a=0$, in this case $\xi_{2}^{1}(t)=$ $\xi_{3}^{1}(t)=\cot (t)-\csc (t)$, so the function (7) becomes

$$
g_{1}(t)=k_{1} \xi_{1}+\bar{k}_{2} \xi_{2}^{1},
$$

where $\bar{k}_{2}=k_{2}+k_{3}$. From Lemma $7(a)$ we obtain that $\mathcal{N}\left(F_{d}, C_{v}\right) \leq 1$. Since now $k_{1}$ and $\bar{k}_{2}$ can be chosen freely, we conclude that $\mathcal{N}\left(F_{d}, C_{v}\right)=1$.

The left system has a center if and only if $b_{22}=-b_{11}$ and $b_{11}^{2}+b_{12} b_{21}<0$. In this case $\delta=1, k_{1}=2 v_{2}, k_{3}=-k_{2}=-u_{1}$, so the function (7) becomes

$$
g_{1}(t)=k_{1} \xi_{1}-2 k_{2} \xi_{2}^{4} \text {. }
$$

From Lemma $6(d)$ we obtain that $\mathcal{N}\left(C_{d}, F_{v}\right) \leq 1$. Rescaling the function $g_{1}$ we see that the parameters $k_{1}$ and $\bar{k}_{2}$ can be chosen freely, hence we conclude that $\mathcal{N}\left(C_{d}, F_{v}\right)=1$.

Finally the lateral systems are centers if and only if $a=0, b_{22}=-b_{11}$ and $b_{11}^{2}+b_{12} b_{21}<0$. In this case the function $(7)$ becomes $g_{1}(t)=k_{1}$. So if $k_{1} \neq 0$, that is $v_{2} \neq 0$, then there is no solutions for the equation $g_{1}(t)=0$; if $k_{1}=0$, that is $v_{2}=0$, then $g_{1}=0$, which implies that all the solutions of system (4) passing through $(0, y)$ for $y>Y_{M}$ are periodic solutions, in other words there are no limit cycles. Hence we conclude that $\mathcal{N}\left(C_{d}, C_{v}\right)=0$.

Proposition 10. The equalities $\mathcal{N}\left(F_{d}, F_{r}\right)=2, \mathcal{N}\left(F_{d}, C_{r}\right)=\mathcal{N}\left(C_{d}, F_{r}\right)=1$ and $\mathcal{N}\left(C_{d}, C_{r}\right)=0$ hold.

Proof. From Proposition $5(c)$ and by a $\Sigma^{+}$-preserving translation we can assume that $a_{11}=a_{22}=a$ with $a \in \mathbb{R}, a_{12}=-a_{21}=1, u_{2}=0$, and $u_{1}<0$ because the right system has a focus which is real for system (4).

In the case that $a<0$ it is easy to see that the focus $\left(-u_{1}, 0\right)$ is an attractor singularity and that the point $\left(0,-a u_{1}\right) \in \Sigma$ is a visible fold point for the right system. So the function $t_{+}(y)$ is defined for every $y<-a u_{1}$. On the other hand its image is the interval $(-\pi, 0)$. Indeed given $y<-a u_{1}$ consider the line $\ell(y)$ passing through the focus point $\left(-u_{1}, 0\right)$ and $(0, y)$. The trajectory of the left system starting at $(0, y)$ returns to the line $\ell(y)$ at $t=-\pi$, so it must return to $\Sigma$ for $t>-\pi$. Thus $t_{+}(y) \in(-\pi, 0)$ for every $y<-a u_{1}$ (see Figure 3.1 right). 

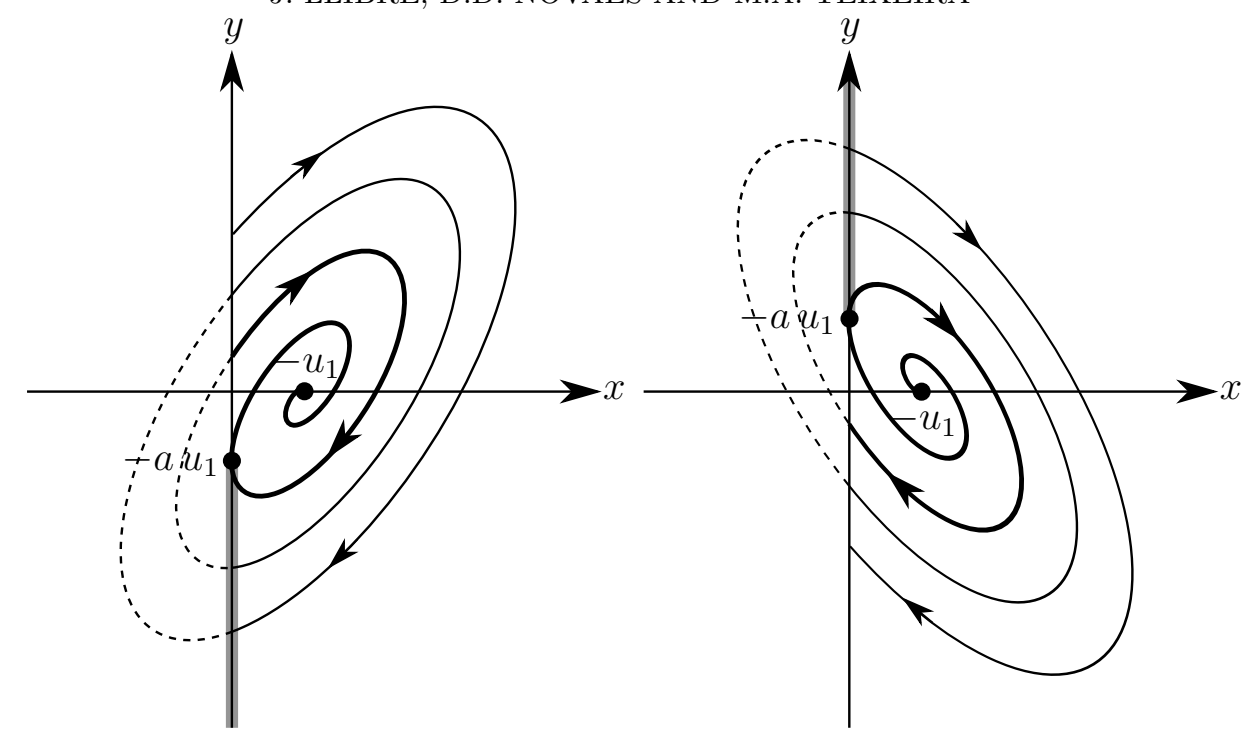

FiguRE 1. Left: Real focus for the right system when $a<0$. In this case the shaded line represents the domain of the definition of the function $t_{+}(y)$. Right: Real focus for the right system when $a>0$. In this case the shaded line represents the domain of the definition of the function $t^{+}(y)$.

In the other case $a>0$ the focus $\left(-u_{1}, 0\right)$ is a repulsive singularity. Considering now the function $t^{+}(y)$ defined for every $y>-a u_{1}$, the same analysis can be done (see Figure 3.1 left).

From now on this proof we assume, without loss of generality, that $a<0$.

We know that $\varphi_{1}^{+}\left(t_{+}(y), 0, y\right)=0$ for every $y<-a u_{1}$, that is

$$
-u_{1}+e^{a t_{+}(y)}\left(u_{1} \cos \left(t_{+}(y)\right)+y \sin \left(t_{+}(y)\right)\right)=0 .
$$

Hence taking $y_{+}=\left.F\right|_{(-\pi, 0)}$ we have that $y_{+}\left(t_{+}(y)\right)=y$ for every $y<-a u_{1}$.

Now we claim that $t_{+}\left(y_{+}(t)\right)=t$ for every $t \in(-\pi, 0)$. Indeed for $t_{0} \in$ $(-\pi, 0)$, let $y_{0}=y_{+}\left(t_{0}\right)$. From Lemma $8(a) y_{0}<-a u_{1}$, so from the above comments we obtain that $y_{0}=y_{+}\left(t_{+}\left(y_{0}\right)\right)$. Thus $y_{+}\left(t_{0}\right)=y_{+}\left(t_{+}\left(y_{0}\right)\right)$. Again from Lemma $8(a) y_{+}(t)=F(t)$ is injective on the interval $(-\pi, 0)$, so $t_{0}=$ $t_{+}\left(y_{0}\right)$. Hence $t_{0}=t_{+}\left(y_{0}\right)=t_{+}\left(y_{+}\left(t_{0}\right)\right)$. Since $t_{0}$ was arbitrarily chosen in $(-\pi, 0)$ we conclude that $t_{+}\left(y_{+}(t)\right)=t$ for every $t \in(-\pi, 0)$. Therefore the function $t_{+}:\left(-\infty,-a u_{1}\right) \rightarrow(-\pi, 0)$ is invertible with inverse equal to $y_{+}:(-\pi, 0) \rightarrow\left(-\infty,-a u_{1}\right)$.

Let $Y_{m}=\min \left\{-a u_{1},-v_{2}\right\}$, so computing the zeros of the function (6) for $y<Y_{m}$ is also equivalent to compute the zeros of the function (7) now for $t \in I \subset(-\pi, 0)$, where $I=t_{+}\left(\left(-\infty, Y_{m}\right)\right)$. Note that if $v_{2} \leq a u_{1}$ then $I=(-\pi, 0)$. 
Applying Lemma $6(a)$ we conclude that $\mathcal{N}\left(F_{d}, F_{r}\right) \leq 2$. Now choosing $a=1, u_{1}=-1 / 2, v_{2}=-2 / 11$, and $b_{i, j}$ for $i, j=1,2$ such that $\delta=10$ we obtain $k_{1}=-2, k_{2}=-1 / 2$, and $k_{3}=5$ which implies that $(7)$ has 2 zeros on $(-\pi, 0)$, namely $t_{1} \approx 1.155$ and $t_{2} \approx 2.301$. Since $y^{+}\left(t_{1}\right) \approx 0.049$ and $y^{+}\left(t_{2}\right) \approx-0.515$, for this choice of coefficients, there exists two limit cycles of system (4) passing respectively through the points $\left(0, y_{+}\left(t_{1}\right)\right)$ and $\left(0, y_{+}\left(t_{2}\right)\right)$.

The equalities $\mathcal{N}\left(F_{d}, C_{r}\right)=\mathcal{N}\left(C_{d}, F_{r}\right)=1$ and $\mathcal{N}\left(C_{d}, C_{r}\right)=0$ follows in a similar way to the proof of Proposition 9. This concludes the proof of this proposition.

Proposition 11. The equalities $\mathcal{N}\left(F_{d}, F_{d}\right)=\mathcal{N}\left(F_{d}, C_{d}\right)=1$ and $\mathcal{N}\left(C_{d}, C_{d}\right)=$ 0 hold.

Proof. Here $u_{1}=0$, because the right system have its focus on the line $\Sigma$. From Proposition $5(c)$ and by a $\Sigma^{+}$-preserving translation we can assume that $a_{11}=a_{22}=a$ with $a \in \mathbb{R}, a_{12}=-a_{21}=1$, and $u_{2}=0$.

The function $t^{+}(y)$ is defined for every $y>0$, because the point $(0,0)$ is a focus for the right system. Moreover we compute $t^{+}(y)=\pi$.

Let $Y_{M}=\max \left\{0,-v_{2}\right\}$, so computing the zeros of the function (5) for $y>Y_{M}$ is equivalent to compute the zeros of the linear function

$$
f_{1}(y)=k_{1}+k_{2} y
$$

for $y>Y_{M}$, where $k_{1}=v_{2}(1+\delta)$ and $k_{2}=\left(\delta-e^{a \pi}\right)$. Hence $\mathcal{N}\left(F_{d}, F_{d}\right) \leq 1$. Nevertheless we can choose coefficients such that $\bar{y}>Y_{M}$ where $\bar{y}=\frac{(1+\bar{\delta}) v_{2}}{e^{a \pi}-\delta}$ is the unique zero of (8).

From here the equalities $\mathcal{N}\left(F_{d}, C_{d}\right)=1$ and $\mathcal{N}\left(C_{d}, C_{d}\right)=0$ follows similarly to the proof of Proposition 9. This concludes the proof of proposition.

Proposition 12. The equalities $\mathcal{N}\left(F_{d}, D N_{v}\right)=2$ and $\mathcal{N}\left(C_{d}, D N_{v}\right)=1$ hold.

Proof. From Proposition $5(b)$ and by a $\Sigma^{+}$-preserving translation, we can assume that $a_{11}=a_{22}=a$ with $|a|>1, a_{12}=a_{21}=1, u_{2}=0$, and $u_{1}>0$, because the right system is a diagonalizable node, which is virtual for system (4).

It is easy to see that the point $\left(0,-a u_{1}\right) \in \Sigma$ is an invisible fold point for the right system.

In the case $a<-1$ the node $\left(-u_{1}, 0\right)$ is an attractor singularity. The stable manifold and the strong stable manifold of the node intersect $\Sigma$ at the points $\left(0, y^{s}\right)$ and $\left(0, y^{s s}\right)$, respectively, where $y^{s}=u_{1}<-a u_{1}$ and $y^{s s}=-u_{1}<u_{1}$. So the function $t^{+}(y)$ is defined for every $y>-a u_{1}$ (see Figure 3.1 right).

In the other case $a>1$ the node $\left(u_{1}, 0\right)$ is an repulsive singularity. The stable manifold and the strong stable manifold of the node intersect $\Sigma$ at the points 


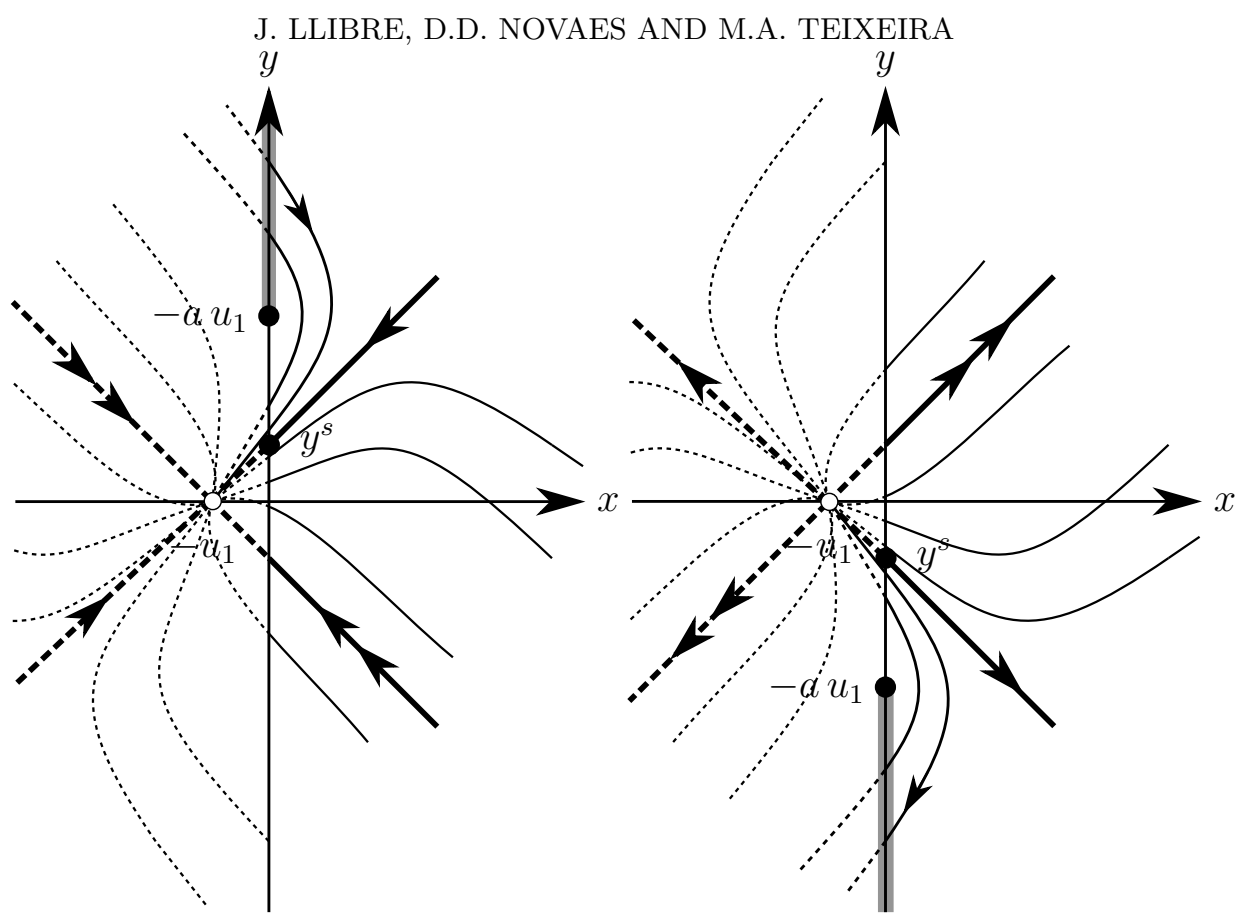

FiguRE 2. Left: Virtual diagonalizable node for the right system when $a<-1$. In this case the shaded line represents the domain of the definition of the function $t^{+}(y)$. Right: Virtual diagonalizable node for the right system when $a>1$. In this case the shaded line represents the domain of the definition of the function $t_{+}(y)$

$\left(0, y^{s}\right)$ and $\left(0, y^{s s}\right)$, respectively, where $y^{s}=-u_{1}>-a u_{1}$ and $y^{s s}=u_{1}>-u_{1}$. So the function $t_{+}(y)$ is defined for every $y<-a u_{1}$ (see Figure 3.1 left).

From now on this proof we assume, without loss of generality, that $a<-1$.

We know that $\varphi_{1}^{+}\left(t^{+}(y), 0, y\right)=0$ for every $y>-a u_{1}$, that is

$$
-u_{1}+e^{a t^{+}(y)}\left(u_{1} \cosh \left(t^{+}(y)\right)+y \sinh \left(t^{+}(y)\right)\right)=0 .
$$

Hence taking $y^{+}=\left.G\right|_{\mathbb{R}^{+}}$we have that $y^{+}\left(t^{+}(y)\right)=y$ for every $y>-a u_{1}$.

The image of the function $t^{+}$is $\mathbb{R}^{+}$. Indeed, computing implicitly the derivative in $y$ of the identity $y^{+}\left(t^{+}(y)\right)=y$ we obtain

$$
\frac{d t^{+}(y)}{d y}=P\left(t^{+}(y)\right), \quad \text { where } \quad P(\theta)=\frac{\sinh (\theta)}{u_{1}\left(\operatorname{csch}(\theta)-e^{-a \theta}(a+\operatorname{coth}(\theta))\right)} .
$$

It is easy to see that $P(\theta)>0$ for every $\theta>0$. So any solution $\theta(y)$ of the differential equation $\dot{\theta}=F(\theta)$ starting at $\theta=\bar{\theta}>0$ and $y=\bar{y}$, i.e. $\theta(\bar{y})=\bar{\theta}$, keeps itself positive for every $y>\bar{y}$, moreover this solution will 
be strictly increasing. Hence we conclude that $t^{+}(y)$ is a positive strictly increasing function for $y>-a u_{1}$.

We claim that $t^{+}\left(y^{+}(t)\right)=t$ for every $t>0$. Indeed for $t_{0}>0$, let $y_{0}=$ $y^{+}\left(t_{0}\right)$. From Lemma $8(b) y_{0}>-a u_{1}$, so from the above comments we obtain that $y_{0}=y^{+}\left(t^{+}\left(y_{0}\right)\right)$. Thus $y^{+}\left(t_{0}\right)=y^{+}\left(t^{+}\left(y_{0}\right)\right)$. Again from Lemma 8 (b) $y^{+}(t)=G(t)$ is injective on $\mathbb{R}^{+}$, so $t_{0}=t^{+}\left(y_{0}\right)$. Hence $t_{0}=t^{+}\left(y_{0}\right)=$ $t^{+}\left(y^{+}\left(t_{0}\right)\right)$. Since $t_{0}>0$ was arbitrarily chosen we conclude that $t^{+}\left(y^{+}(t)\right)=t$ for every $t>0$. Therefore the function $t^{+}:\left(-a u_{1}, \infty\right) \rightarrow \mathbb{R}^{+}$is invertible with inverse equal to $y^{+}: \mathbb{R}^{+} \rightarrow\left(-a u_{1}, \infty\right)$.

Computing the zeros of the function (5) for $y>Y_{M}$ is equivalent to compute the zeros of the function

$$
g_{2}(t)=f\left(y^{+}(t)\right)=k_{1} \xi_{1}+k_{2} \xi_{2}^{2}+k_{3} \xi_{3}^{2}
$$

for $t \in I \subset \mathbb{R}^{+}$, where $k_{1}=v_{2}(1+\delta), k_{2}=u_{1}, k_{3}=-\delta u_{1}, \delta=e^{-\frac{\left(b_{11}+b_{22}\right) \pi}{\Gamma}}$, and here $I=t^{+}\left(\left(Y_{M}, \infty\right)\right)$. Note that if $v_{2} \geq a u_{1}$ then $I=\mathbb{R}^{+}$.

Applying Lemma $6(b)$ we conclude that $\mathcal{N}\left(F_{d}, D N_{v}\right) \leq 2$. Now choosing $a=2, u_{1}=10, v_{2}=275 / 16$, and $b_{i, j}$ for $i, j=1,2$ such that $\delta=15$ we obtain $k_{1}=275, k_{2}=10$, and $k_{3}=-150$ which implies that (9) has 2 zeros on $\mathbb{R}^{+}$, namely $t_{1} \approx 0.263$ and $t_{2} \approx 1.838$. Since $y^{+}\left(t_{1}\right) \approx-16.687$ and $y^{+}\left(t_{2}\right) \approx-10.437$, for this choice of coefficients, there exists two limit cycles of system (4) passing respectively through the points $\left(0, y^{+}\left(t_{1}\right)\right)$ and $\left(0, y^{+}\left(t_{2}\right)\right)$.

From here the equality $\mathcal{N}\left(C_{d}, D N_{v}\right)=1$ follows similar to the proof of Proposition 9. This completes the proof of proposition.

Proposition 13. The equalities $\mathcal{N}\left(F_{d}, n D N_{v}\right)=2$ and $\mathcal{N}\left(C_{d}, n D N_{v}\right)=1$ hold.

Proof. From Proposition $5(d)$ and by a $\Sigma^{+}$-preserving translation, we can assume that $a_{11}=a_{12}=a_{22}=\lambda$ with $\lambda= \pm 1, a_{21}=0, u_{2}=0$, and $u_{1}>0$, because the right system is a non diagonalizable node, which is virtual for system (4).

It is easy to see that for $\lambda= \pm 1$ the point $\left(0,-u_{1}\right) \in \Sigma$ is a invisible fold point for the right system and that the invariant manifold of the node intersects $\Sigma$ at the origin $(0,0)$. In order to fix the clockwise orientation of the flow of system (4) we assume that $\lambda=1$, otherwise the first return map would not be defined and there would not exist limit cycles. In this case the function $t_{+}(y)$ is defined for every $y<-u_{1}$.

We know that $\varphi_{1}^{+}\left(t_{+}(y), 0, y\right)=0$ for every $y<-u_{1}$, that is

$$
-u_{1}+e^{t_{+}(y)}\left(u_{1}+y t\right)=0 .
$$

Hence taking $y_{+}=\left.H\right|_{\mathbb{R}^{-}}$we have that $y_{+}\left(t_{+}(y)\right)=y$ for every $y<-u_{1}$. 


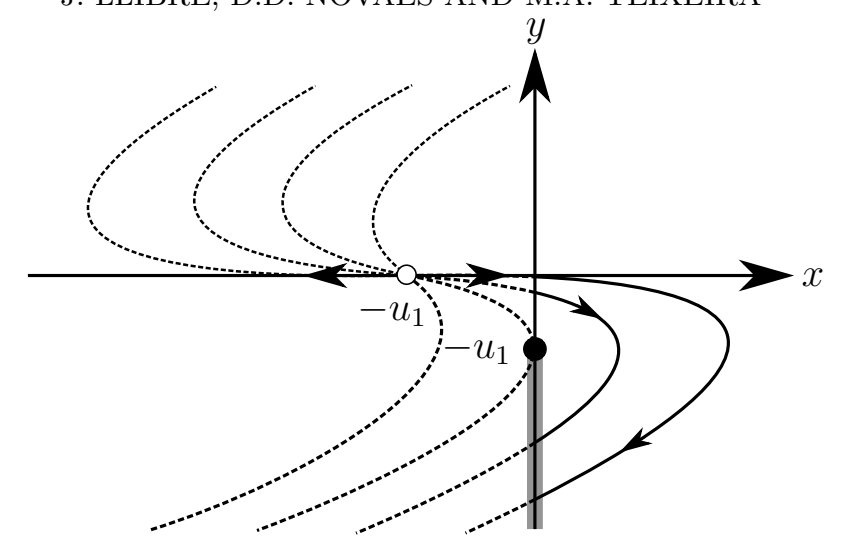

Figure 3. Virtual non-diagonalizable node for the right system when $\lambda=1$. In this case the shaded line represents the domain of the definition of the function $t_{+}(y)$.

The image of the function $t_{+}$is $\mathbb{R}^{+}$. Indeed, computing implicitly the derivative in $y$ of the identity $y_{+}\left(t_{+}(y)\right)=y$ we obtain

$$
\frac{d t_{+}(y)}{d y}=Q\left(t_{+}(y)\right), \quad \text { where } \quad Q(\theta)=\frac{e^{\theta} \theta^{2}}{u_{1}\left(e^{\theta}-\theta-1\right)} .
$$

So the function $t_{+}$is the solution $\theta(y)$ of the above differential equation such that $\theta\left(-u_{1}\right)=0$. It is easy to see that $Q(\theta)>0$, moreover by continuity we have that $Q(0)=2$. So it follows that the solution $\theta(y)$ is strictly increasing. Hence we conclude that $t_{+}(y)$ is strictly increasing function such that $t_{+}\left(-u_{1}\right)=0$, which implies that $t_{+}(y)<0$ for $y<0$.

Now we claim that $t_{+}\left(y_{+}(t)\right)=t$ for every $t<0$. Indeed for $t_{0}<0$, let $y_{0}=y_{+}\left(t_{0}\right)$. From Lemma $8(c) y_{0}<-u_{1}$, so from the above comments we obtain that $y_{0}=y_{+}\left(t_{+}\left(y_{0}\right)\right)$. Thus $y_{+}\left(t_{0}\right)=y_{+}\left(t_{+}\left(y_{0}\right)\right)$. Again from Lemma $8(c)$ the function $y_{+}(t)=H(t)$ is injective, so $t_{0}=t_{+}\left(y_{0}\right)$. Hence $t_{0}=t_{+}\left(y_{0}\right)=t_{+}\left(y_{+}\left(t_{0}\right)\right)$. Since $t_{0}>0$ is arbitrarily chosen we conclude that $t_{+}\left(y_{+}(t)\right)=t$ for every $t>0$. Therefore the function $t_{+}:\left(-\infty,-u_{1}\right) \rightarrow \mathbb{R}^{-}$is invertible with inverse equal to $y_{+}: \mathbb{R}^{-} \rightarrow\left(\infty,-u_{1}\right)$.

Let $Y_{m}=\min \left\{-u_{1},-v_{2}\right\}$, so computing the zeros of the function (6) for $y<Y_{m}$ is equivalent to compute the zeros of the function

$$
g_{3}(t)=f\left(y_{+}(t)\right)=k_{1} \xi_{1}+k_{2} \xi_{2}^{3}+k_{3} \xi_{3}^{3}
$$

for $t \in I \subset \mathbb{R}^{-}$, where $k_{1}=v_{2}(1+\delta), k_{2}=u_{1}, k_{3}=-\delta u_{1}, \delta=e^{-\frac{\left(b_{11}+b_{22}\right) \pi}{\Gamma}}$, and here $I=y_{-}\left(\left(-\infty, Y_{M}\right)\right)$. Note that if $v_{2} \geq u_{1}$ then $I=\mathbb{R}^{-}$.

Applying Lemma $6(c)$ we conclude that $\mathcal{N}\left(F_{d}, n D N_{v}\right) \leq 2$. Now choosing $u_{1}=3 / 2, v_{2}=90 / 103 \approx 0.874$, and $b_{i, j}$ for $i, j=1,2$ such that $\delta=100 / 3$ we obtain $k_{1}=30, k_{2}=3 / 2$, and $k_{3}=-50$ which implies that $(7)$ has 2 zeros 
on $\mathbb{R}^{+}$, namely $t_{1} \approx 1.422$ and $t_{2} \approx 3.788$. Since $y^{+}\left(t_{1}\right) \approx-0.800>-v_{2}$ and $y^{+}\left(t_{2}\right) \approx-0,387>-v_{2}$, for this choice of coefficients, there exists two limit cycles of system (4) passing respectively through the points $\left(0, y^{+}\left(t_{1}\right)\right)$ and $\left(0, y^{+}\left(t_{2}\right)\right)$.

From here applying Lemma $7(b)$ the equality $\mathcal{N}\left(C_{d}, n D N_{v}\right)=1$ follows similar to the proof of Proposition 9. This concludes the proof of proposition.

Proposition 14. The equalities $\mathcal{N}\left(F_{d}, S_{r}\right)=2, \mathcal{N}\left(F_{d}, S_{r}^{0}\right)=\mathcal{N}\left(C_{d}, S_{r}\right)=1$ and $\mathcal{N}\left(C_{d}, S_{r}^{0}\right)=0$ hold.

Proof. From Proposition $5(a)$ and by a $\Sigma^{+}$-preserving translation, we can assume that $a_{11}=a_{22}=a$ with $|a|<1, a_{12}=a_{21}=1, u_{2}=0$, and $u_{1}<0$, because the right system is a saddle, which is real for system (4).

It is easy to see that the point $\left(0,-a u_{1}\right) \in \Sigma$ is an invisible fold point for the right system and that the stable and unstable invariant manifolds of the saddle intersect $\Sigma$ at the points $\left(0, y^{s}\right)$ and $\left(0, y^{u}\right)$, respectively, where $y^{s}=u_{1}$ and $y_{u}=-u_{1}$. So the function $t^{+}(y)$ is defined for every $-a u_{1}<y<u_{1}$.

We know that $\varphi_{1}^{+}\left(t^{+}(y), 0, y\right)=0$ for every $-a u_{1}<y<u_{1}$, that is

$$
-u_{1}+e^{a t^{+}(y)}\left(u_{1} \cosh \left(t^{+}(y)\right)+y \sinh \left(t^{+}(y)\right)\right)=0 .
$$

Hence taking $y^{+}=\left.G\right|_{\mathbb{R}^{+}}$we have that $y^{+}\left(t^{+}(y)\right)=y$ for every $-a u_{1}<y<u_{1}$.

In the proof of Proposition 12 we have seen that the function $t^{+}:\left(-a u_{1}, \infty\right) \rightarrow$ $\mathbb{R}^{+}$is invertible with inverse equal to $y^{+}: \mathbb{R}^{+} \rightarrow\left(-a u_{1}, \infty\right)$. So its restriction to $-a u_{1}<y<u_{1}$ is also invertible with inverse defined in $t^{+}\left(-a u_{1}, u_{1}\right)$.

Computing the zeros of the function (5) for $Y_{M}<y<u_{1}$ is equivalent to compute the zeros of the function (9) for $t \in I \subset \mathbb{R}^{+}$, where $k_{1}=v_{2}(1+\delta)$, $k_{2}=u_{1}, k_{3}=-\delta u_{1}, \delta=e^{-\frac{\left(b_{11}+b_{22}\right) \pi}{\Gamma}}$, and $I=t^{+}\left(\left(Y_{M},-u_{1}\right)\right)$.

Applying Lemma $6(b)$ we conclude that $\mathcal{N}\left(F_{d}, S_{r}\right) \leq 2$. Now choosing $a=-1 / 2, u_{1}=-100, v_{2}=1600 / 127$, and $b_{i, j}$ for $i, j=1,2$ such that $\delta=7 / 20$ we obtain $k_{1}=80, k_{2}=-100$, and $k_{3}=35$ which implies that (9) has 2 zeros on $I_{5}$, namely $t_{1} \approx 0.689$ and $t_{2} \approx 2.761$. Since $y^{+}\left(t_{1}\right) \approx-22.071 \in(-50,100)$ and $y^{+}\left(t_{2}\right) \approx 50.318 \in(-50,100)$, for this choice of coefficients, there exists two limit cycles of system (4) passing respectively through the points $\left(0, y^{+}\left(t_{1}\right)\right)$ and $\left(0, y^{+}\left(t_{2}\right)\right)$. This concludes the proof of proposition.

The right system has a saddle with trace equal 0 if and only if $a=0$, in this case $\xi_{2}^{2}(t)=\xi_{3}^{2}(t)=\operatorname{coth}(t)-\operatorname{csch}(t)$. From here the equalities $\mathcal{N}\left(F_{d}, S_{r}^{0}\right)=1$, $\mathcal{N}\left(C_{d}, S_{r}\right)=1$ and $\mathcal{N}\left(C_{d}, S_{r}^{0}\right)=0$ follows as in the proof of Proposition 9. It concludes the proof of this proposition. 
3.2. Left system has a saddle with trace equal 0 . In this case $b_{22}=-b_{11}$, $b_{11}^{2}+b_{12} b_{21}>0$ and $v_{1}>0$ and the point $\left(-v_{1},-v_{2}\right)$ is a singularity of saddle type.

Let $\Gamma=\sqrt{b_{11}^{2}+b_{12} b_{21}}$, let $y^{u}$ be the $y$-coordinate of the intersection between the unstable manifold with $\Sigma$, and let $y^{s}$ be the $y$-coordinate of the intersection between the stable manifold with $\Sigma$. We compute

$$
y^{u}=-v_{2}+\frac{v_{1}\left(\Gamma-b_{11}\right)}{b_{12}} \text { and } y^{s}=-v_{2}-\frac{v_{1}\left(\Gamma+b_{11}\right)}{b_{12}} .
$$

In order to fix the clockwise orientation of the flow of system (4) we assume that $y^{s}<y^{s}$, which is equivalent to assume that $b_{12}>0$.

The left system has an invisible fold point $(0, \check{y})$ given by

$$
\check{y}=-v_{2}-\frac{b_{11} v_{1}}{b_{12}} \text {. }
$$

For $y^{s}<y<y^{y}$ we define

$$
t^{*}(y)=\frac{1}{\Gamma} \log \left(\frac{v_{1}\left(\Gamma-b_{11}\right)-b_{12}\left(v_{2}+y\right)}{v_{1}\left(\Gamma+b_{11}\right)+b_{12}\left(v_{2}+y\right)}\right) .
$$

So $t^{-}(y)=t^{*}(y)<0$ for $\check{y}<y<y^{u}$ and $t^{-}(y)=t^{*}(y)>0$ for $y^{s}<y<\check{y}$.

Proposition 15. The equalities $\mathcal{N}\left(S_{r}^{0}, F_{v}\right)=1$ and $\mathcal{N}\left(S_{r}^{0}, C_{v}\right)=0$ holds.

Proof. From Proposition 5 (c) we can assume that $a_{11}=a_{22}=a$ with $a \in \mathbb{R}$, $a_{12}=-a_{21}=1$, and by a $\Sigma^{+}$-preserving translation we can take $u_{2}=0$. Moreover $u_{1}>0$ because the right system has a focus which is virtual for system (4).

From the proof of Proposition 9 we know that the function $t^{+}:\left(-a u_{1}, \infty\right) \rightarrow$ $(0, \pi)$, such that $\varphi^{+}\left(t^{+}(y), 0, y\right)=0$ for $y>-a u_{1}$, is invertible with inverse equal to $y^{+}=F:(0, \pi) \rightarrow\left(-a u_{1}, \infty\right)$.

Let $Y_{M}=\max \left\{-a u_{1}, \check{y}\right\}$, so computing the zeros of the function (5) for $Y_{M}<y<y^{u}$ is equivalent to compute the zeros of the function

$$
g_{4}(t)=f\left(y^{+}(t)\right)=k_{1} \xi_{1}+k_{2} \xi_{2}^{4}
$$

for $t \in I \subset(0, \pi)$, where $k_{1}=2 \frac{b_{11} v_{1}+b_{12} v_{2}}{b_{12}}$ and $k_{2}=-2 u_{1}$, and here $I=t^{+}\left(\left(Y_{M}, y^{u}\right)\right)$.

Applying Lemma $6(d)$ we obtain that $\mathcal{N}\left(S_{r}^{0}, F_{v}\right) \leq 1$. Rescaling the function $g_{4}(t)$ we can see that the parameters $k_{1}$ and $k_{2}$ can be chosen freely, so we conclude that $\mathcal{N}\left(S_{r}^{0}, F_{v}\right)=1$.

The right system has a center if and only if $a=0$. In this case $\xi_{2}^{4}=0$ and the function (10) becomes $g_{4}(t)=k_{1}$. So if $k_{1} \neq 0$, that is $b_{11} v_{1} \neq-b_{12} v_{2}$, then there are no solutions for the equation $g_{4}(t)=0$; and if $k_{1}=0$, that is 
$b_{11} v_{1}=-b_{12} v_{2}$, then $g_{4}=0$, that is system (4) is a center. Hence we conclude that $\mathcal{N}\left(S_{r}^{0}, C_{v}\right)=0$.

Proposition 16. The equalities $\mathcal{N}\left(S_{r}^{0}, F_{r}\right)=1$ and $\mathcal{N}\left(S_{r}^{0}, C_{r}\right)=0$ hold.

Proof. From Proposition 5 (c) we can assume that $a_{11}=a_{22}=a$ with $a \in \mathbb{R}$, $a_{12}=-a_{21}=1$, and by a $\Sigma^{+}$-preserving translation we can take $u_{2}=0$. Moreover $u_{1}<0$ because the right system has a focus which is real for system 4 .

From the proof of Proposition 10 we know that the function $t_{+}:\left(-\infty,-a u_{1}\right) \rightarrow$ $(-\pi, 0)$ is invertible with inverse equal to $y_{+}=F:(-\pi, 0) \rightarrow\left(-\infty,-a u_{1}\right)$. Here as we have done in the proof of Proposition 10 we are assuming, without loss of generality, that $a<0$.

Let $Y_{m}=\min \left\{-a u_{1}, \check{y}\right\}$, so computing the zeros of the function (6) for $y^{s}<y<Y_{m}$ is also equivalent to compute the zeros of the function (10) now for $t \in I \subset(-\pi, 0)$, where $I=t_{+}\left(\left(y^{s}, Y_{m}\right)\right)$.

Applying Lemma $6(d)$ we obtain that $\mathcal{N}\left(S_{r}^{0}, F_{r}\right) \leq 1$. Rescaling the function $g_{4}(t)$ we can see that the parameters $k_{1}$ and $k_{2}$ can be chosen freely, so we conclude that $\mathcal{N}\left(S_{r}^{0}, F_{v}\right)=1$.

From here the equality $\mathcal{N}\left(S_{r}^{0}, C_{r}\right)=0$ follows similar to the proof of Proposition 16. It concludes the proof of this proposition.

Proposition 17. The equality $\mathcal{N}\left(S_{r}^{0}, D N_{v}\right)=1$ holds.

Proof. From Proposition $5(b)$ and by a $\Sigma^{+}$-preserving translation, we can assume that $a_{11}=a_{22}=a$ with $|a|>1, a_{12}=a_{21}=1, u_{2}=0$, and $u_{1}>0$, because the right system has a diagonalizable node, which is virtual for system (4).

Following the proof of Proposition 12 the function $t^{+}:\left(-a u_{1}, \infty\right) \rightarrow \mathbb{R}^{+}$is invertible with inverse equal to $y^{+}=G: \mathbb{R}^{+} \rightarrow\left(-a u_{1}, \infty\right)$. Here as we have done in the proof of Proposition 11 we are assuming, without loss of generality, that $a<1$.

Let $Y_{M}=\max \left\{-a u_{1}, \check{y}\right\}$, so computing the zeros of the function (5) for $Y_{M}<y<y^{u}$ is equivalent to compute the zeros of the function

$$
g_{5}(t)=f\left(y^{+}(t)\right)=k_{1} \xi_{1}+k_{2} \xi_{2}^{5}
$$

for $t \in I \subset \mathbb{R}^{+}$, where $k_{1}=\frac{2 b_{11} v_{1}}{b_{12}}+2 v_{2}, k_{2}=-2 u_{1}$, and $I=y^{+}\left(\left(Y_{M}, y^{u}\right)\right)$.

From here, applying Lemma $6(e)$ we conclude that $\mathcal{N}\left(S_{r}^{0}, D N_{v}\right) \leq 1$. Rescaling the function $g_{5}(t)$ we see that the parameters $k_{1}$ and $k_{2}$ can be chosen freely, so we conclude that $\mathcal{N}\left(S_{r}^{0}, D N_{v}\right)=1$.

Proposition 18. The equality $\mathcal{N}\left(S_{r}^{0}, n D N_{v}\right)=1$ holds. 
Proof. From Proposition 5 and by a $\Sigma^{+}$-preserving translation, we can assume that $a_{11}=a_{12}=a_{22}=\lambda$ with $\lambda= \pm 1, a_{21}=0, u_{2}=0$, and $u_{1}>0$, because the right system has a non diagonalizable node, which is virtual for system (4).

Following the proof of Proposition $13(d)$ the function $t^{+}:\left(-u_{1}, \infty\right) \rightarrow \mathbb{R}^{+}$ is invertible with inverse equal to $y^{+}=H: \mathbb{R}^{+} \rightarrow\left(-u_{1}, \infty\right)$. Here as we have done in the proof of Proposition 13 we are assuming, without loss of generality, that $\lambda=1$.

Let $Y_{M}=\max \left\{-u_{1}, \check{y}\right\}$, so computing the zeros of the function (5) for $Y_{M}<y<y^{u}$ is equivalent to compute the zeros of the function

$$
g_{6}(t)=f\left(y^{+}(t)\right)=k_{1} \xi_{1}+k_{2} \xi_{2}^{6}
$$

for $t \in I \subset \mathbb{R}^{+}$, where $k_{1}=\frac{2 b_{11} v_{1}}{b_{12}}+2 v_{2}, k_{2}=-2 u_{1}$, and $I=y^{+}\left(\left(Y_{M}, y^{u}\right)\right)$.

Applying Lemma $6(f)$ we conclude that $\mathcal{N}\left(S_{r}^{0}, n D N_{v}\right) \leq 1$. Rescaling the function $g_{6}(t)$ we see that the parameters $k_{1}$ and $k_{2}$ can be chosen freely, so we conclude that $\mathcal{N}\left(S_{r}^{0}, n D N_{v}\right)=1$.

Proposition 19. The equalities $\mathcal{N}\left(S_{r}^{0}, S_{r}\right)=1$ and $\mathcal{N}\left(S_{r}^{0}, S_{r}^{0}\right)=0$ hold.

Proof. From Proposition 5 and by a $\Sigma^{+}$-preserving translation, we can assume that $a_{11}=a_{22}=a$ with $|a|<1, a_{12}=a_{21}=1, u_{2}=0$, and $u_{1}<0$, because the right system has a saddle, which is real for system (4).

Following the proof of Proposition $14(a)$ the function $t^{+}:\left(-a u_{1}, \infty\right) \rightarrow \mathbb{R}^{+}$ is invertible with inverse equal to $y^{+}=G: I_{5} \rightarrow\left(-a u_{1}, u_{1}\right)$ where $I_{5}=$ $t^{+}\left(-a u_{1},-u_{1}\right)$.

Let $Y_{M}=\max \left\{-a u_{1}, \check{y}\right\}$ and $Y_{m}=\min \left\{u_{1}, y^{u}\right\}$, so computing the zeros of the function (5) for $Y_{M}<y<Y_{m}$ is equivalent to compute the zeros of the function (11) for $t \in I \subset \mathbb{R}^{+}$, where $k_{1}=\frac{2 b_{11} v_{1}}{b_{12}}+2 v_{2}$ and $k_{2}=-2 u_{1}$.

From here, applying Lemma $6(e)$ we conclude that $\mathcal{N}\left(S_{r}^{0}, S_{r}\right) \leq 1$. Rescaling the parameters of the function (11) we see that the parameters $k_{1}$ and $k_{2}$ can be chosen freely, so we conclude that $\mathcal{N}\left(S_{r}^{0}, S_{r}\right)=1$.

The right system has a saddle with trace equal 0 if and only if $a=0$. In this case $\xi_{2}^{5}=0$ and the function (11) becomes $g_{5}(t)=k_{1}$. So if $k_{1} \neq 0$, that is $b_{11} v_{1} \neq 0$, then there are no solutions for the equation $g_{5}(t)=0$; and if $k_{1}=0$, that is $b_{11} v_{1}=0$, then $g_{5}=0$, which implies that all the solutions of system (4) passing through $(0, y)$ for $Y_{M}<y<Y_{m}$ are periodic solutions, in other words there are no limit cycles. Hence we conclude that $\mathcal{N}\left(S_{r}^{0}, S_{r}^{0}\right)=0$. 
3.3. Left system has a virtual or real center. In this case $v_{1} \neq 0, b_{11}=$ $-b_{22}, b_{11}^{2}+b_{12} b_{21}<0$ and the point $\left(-v_{1},-v_{2}\right)$ is a singularity of center type.

The left system has a fold point $(0, \check{y})$ given by

$$
\check{y}=-v_{2}-\frac{b_{11} v_{1}}{b_{12}} \text {. }
$$

which is visible if $v_{1}>0$, and invisible if $v_{1}<0$.

Let $\Gamma=2 \sqrt{-b_{11}^{2}-b_{12} b_{21}}$. The function $t^{-}(y)$ is defined for every $y>\check{y}$, and we compute

$$
t^{-}(y)=\frac{4}{\Gamma}\left(\arctan \left(\frac{2\left(b_{11} v_{1}+b_{12}\left(v_{2}+y\right)\right)}{v_{1} \Gamma}\right)-\pi\right) .
$$

Analogously, the function $t_{-}(y)$ is defined for every $y<\check{y}$, and we compute

$$
t_{-}(y)=\frac{4}{\Gamma}\left(\arctan \left(\frac{2\left(b_{11} v_{1}+b_{12}\left(v_{2}+y\right)\right)}{v_{1} \Gamma}\right)+\pi\right) .
$$

In order to fix the clockwise orientation of the flow of system (4) we assume that $Y_{1}\left(-v 1,1-v_{2}\right)=b_{12}>0$.

Proposition 20. The equalities $\mathcal{N}\left(C, F_{v}\right)=\mathcal{N}\left(C, F_{r}\right)=1$ and $\mathcal{N}\left(C, C_{v}\right)=$ $\mathcal{N}\left(C, C_{v}\right)=0$ hold.

Proof. In Corollary 3 these equalities have already been proved when the left system has a center in $\Sigma$. So we can take $v_{1} \neq 0$.

To prove $\mathcal{N}\left(C, F_{v}\right)=1$ we follow the proof of Proposition (9) and then we compute the solutions of the function $(5)$ for $y>Y_{M}=\max \left\{\check{y},-a u_{1}\right\}$. To prove $\mathcal{N}\left(C, F_{r}\right)=1$ we follow the proof of Proposition 10 and then we compute the solutions of the function (6) for $y<Y_{m}=\min \left\{\check{y},-a u_{1}\right\}$. In both case the equations to solve are equivalent to

$$
k_{1}+k_{2} \xi_{2}^{4}=0
$$

where $k_{1}=\left(b_{11} v_{1}+b_{12} v_{2}\right) / b_{12}$ and $k_{2}=-u_{1}$. So applying Lemma $6(d)$ we conclude that $\mathcal{N}\left(C, F_{v}\right)=\mathcal{N}\left(C, F_{r}\right)=1$.

From here the equality $\mathcal{N}\left(C, C_{v}\right)=\mathcal{N}\left(C, C_{v}\right)=0$ follows similar to the proof of Proposition 15. It concludes the proof of this proposition.

Proposition 21. The equalities $\mathcal{N}\left(C, F_{d}\right)=1$ and $\mathcal{N}\left(C, C_{d}\right)=0$ hold.

Proof. In Corollary 3 these equalities have already been proved when the left system has a center in $\Sigma$. So we can take $v_{1} \neq 0$.

Following the proof of Proposition (9) we compute the solutions of the function (5) for $y>Y_{M}=\max \{\check{y}, 0\}$, which is equivalent to compute the zeros of the following equation

$$
k_{1}+k_{2} y=0,
$$


where $k_{1}=2\left(b_{11} v_{1}+b_{12} v_{2}\right) / b_{12}$ and $k_{2}=\left(1-e^{a \pi}\right)$. So analogously to the proof of Proposition (11) we conclude that $\mathcal{N}\left(C, F_{v}\right)=1$.

From here the equalities $\mathcal{N}\left(C, F_{d}\right)=1$ and $\mathcal{N}\left(C, C_{d}\right)=0$ follows similar to the proof of Proposition 9. It concludes the proof of this proposition.

Proposition 22. The equalities $\mathcal{N}\left(C, D N_{v}\right)=\mathcal{N}\left(C, S_{r}\right)=1$ and $\mathcal{N}\left(C, S_{r}^{0}\right)=$ 0 hold.

Proof. In Corollary 3 these equalities have already been proved when the left system has a center in $\Sigma$. So we can take $v_{1} \neq 0$.

To prove the equality $\mathcal{N}\left(C, D N_{v}\right)=1$ we follow the proof of Proposition (12), then we compute the solutions of the function (5) for $y>Y_{M}=$ $\max \left\{\check{y},-a u_{1}\right\}$. To prove the equality $\mathcal{N}\left(C, S_{r}\right)=1$ we follow the proof of Proposition 14, then we compute the solutions of the function (5) for $Y_{M}<y<u_{1}$. In both case the equations to solve are equivalent to

$$
k_{1}+k_{2} \xi_{2}^{5}=0
$$

where $k_{1}=2\left(b_{11} v_{1}+b_{12} v_{2}\right) / b_{12}$ and $k_{2}=-2 u_{1}$. From here the proof of the equality $\mathcal{N}\left(C, D N_{v}\right)=\mathcal{N}\left(C, S_{r}\right)=1$ follows similar to the proof of the Propositions 17 and 19 , and the equality $\mathcal{N}\left(C, S_{r}^{0}\right)=0$ follows similar to the proof of Proposition (19). It concludes the proof of this proposition.

Proposition 23. The equality $\mathcal{N}\left(C, n D N_{v}\right)=1$ holds.

Proof. In Corollary 3 this equality has already been proved when the left system has a center in $\Sigma$. So we can take $v_{1} \neq 0$.

Following the proof of Proposition (13) we compute the solutions of the function (6) for $y<Y_{m}=\left\{\check{y},-u_{1}\right\}$, which is equivalent to compute the zeros of the following equation

$$
k_{1}+k_{2} \xi_{2}^{6}=0
$$

where $k_{1}=\left(b_{11} v_{1}+b_{12} v_{2}\right) / b_{12}$ and $k_{2}=-u_{1}$. So analogously to the proof of Proposition (18) we conclude that $\mathcal{N}\left(C, n D N_{v}\right)=1$. It concludes the proof of this proposition.

\section{ACKNOWLEDGEMENTS}

The first author is partially supported by a MINECO/FEDER grant MTM20 08-03437 and MTM2013-40998-P, an AGAUR grant number 2013SGR-568, an ICREA Academia, the grants FP7-PEOPLE-2012-IRSES 318999 and 316338, and a FEDER-UNAB 10-4E-378. The second author is partially supported by a FAPESP-BRAZIL grant 2013/16492-0. The third authors is partially supported by a FAPESP-BRAZIL grant 2012/18780-0. The three authors are also supported by a CAPES CSF-PVE grant 88881.030454/ 2013-01 from the program CSF-PVE. 


\section{REFERENCES}

[1] A. Andronov, A. Vitt and S. Khaikin, Theory of Oscillations, Pergamon Press, Oxford, 1966.

[2] J. C. Artés, J. Llibre, J. C. Medrado and M. A. Teixeira, Piecewise linear differential systems with two real saddles, Mathematics and Computers in Simulation 95 (2013), 13-22.

[3] A. D. BAzYkin, Nonlinear Dynamics of Interacting Populations, River-Edge, NJ: World Scientific, 1998.

[4] E. A. Barbashin, Introduction to the Theory of Stability (T. Lukes, Ed.), Noordhoff, Groningen, 1970.

[5] D. Bothe, Periodic solutions of non-smooth friction oscillators, Z. Angew. Math. Phys. 50 (1999), 779808.

[6] D.C. Braga And L.F. Mello, More than Three Limit Cycles in Discontinuous Piecewise Linear Differential Systems with Two Zones in the Plane, Int. J. Bifurc. Chaos 24 (2014), 1450056 (10 pages).

[7] B. Brogliato, Nonsmooth Mechanics, New York: Springer-Verlag, 1999.

[8] C. Buzzi, C. Pessoa And J. Torregrosa, Piecewise linear perturbations of a linear center, Discrete Contin. Dyn. Syst. 33 (2013), 3915-3936.

[9] S. Coombes, Neuronal networks with gap junctions: A study of piecewise linear planar neuron models, SIAM Applied Mathematics 7 (2008), 1101-1129.

[10] D. De Carvalho Braga and L. F. Mello, Limit cycles in a family of discontinuous piecewise linear differential systems with two zones in the plane Nonlinear Dynam. 73 (2013), 1283-1288.

[11] M. di Bernardo, C. J. Budd, A. R. Champneys, P. Kowalczyk, PiecewiseSmooth Dynamical Systems: Theory and Applications, Appl. Math. Sci. Series 163, Springer-Verlag, London, 2008.

[12] R.D. EuzÉBIO AND J. LliBRE, On the number of limit cycles in discontinuous piecewise linear differential systems with two pieces separated by a straight line, to appear in J. Math. Anal. Appl., 2014.

[13] E. Freire, E. Ponce, F. Rodrigo And F. Torres, Bifurcation sets of continuous piecewise linear systems with two zones, Int. J. Bifurcation and Chaos 8 (1998), 20732097.

[14] F. Giannakopoulos And K. Pliete, Planar systems of piecewise linear differential equations with a line of discontinuity, Nonlinearity 14 (2001), 1611-1632.

[15] M. HAN AND W. Zhang, On Hopf bifurcation in non-smooth planar systems, J. of Differential Equations 248 (2010), 2399-2416.

[16] C. Henry, Differential equations with discontinuous righthand side for planning procedure, J. Econom. Theory 4 (1972), 541-551.

[17] S. M. HuAN AND X. S. YANG, The number of limit cycles in general planar piecewise linear systems, Discrete and Continuous Dynamical Systems-A 32 (2012), 2147-2164.

[18] S. M. HuAN AND X. S. YANG, Existence of limit cycles in general planar piecewise linear systems of saddle-saddle dynamics, Nonlinear Anal. 92 (2013), 82-95.

[19] S. M. HuAN AND X. S. YANG, On the number of limit cycles in general planar piecewise linear systems of node-node types, J. Math. Anal. Appl. 411 (2014), 340-353.

[20] Y. Ilyashenko, Centennial history of Hilbert's 16th problem, Bull. Amer. Math. Soc. 39 (2002), 301-354.

[21] T. Iто A Filippov solution of a system of differential equations with discontinuous right-hand sides, Economic Letters 4 (1979), 349-354. 
[22] S.J. Karlin And W.J. Studden, T-Systems: With Applications in Analysis and Statistics, Pure Appl. Math., Interscience Publishers, New York, London, Sidney, 1966.

[23] V. KRIVAn, On the Gause predator-prey model with a refuge: A fresh look at the history, Journal of Theoretical Biology 274 (2011), 67-73.

[24] M. KUnZE AND T. KÜPPER, Qualitative bifurcation analysis of a non-smooth frictionoscillator model, Z. Angew. Math. Phys. 48 (1997), 7-101.

[25] R. E. LEINE AND D. H. VAN CAMPEN, Discontinuous bifurcations of periodic solutions, Mathematical and Computing Modelling 36 (2002), 259-273.

[26] J. Llibre, D.D. Novaes And M.A. TeixeIRA, Limit cycles bifurcating from the periodic orbits of a discontinuous piecewise linear differential center with two zones, preprint, 2015.

[27] J. Llibre AND E. Ponce, Three nested limit cycles in discontinuous piecewise linear differential systems with two zones, Dynamics of Continuous, Discrete \& Impulsive Systems, Series B 19 (2012), 325-335.

[28] J. Llibre, M.A. TeiXeira And J. Torregrosa, Lower bounds for the maximum number of limit cycles of discontinuous piecewise linear differential systems with a straight line of separation, Int. J. Bifurcation and Chaos 23 (2013), 1350066-10 pp.

[29] R. Lum, L. O. ChuA, Global properties of continuous piecewise-linear vector fields. Part I: Simplest case in $R^{2}$, Memorandum UCB/ERL M90/22, University of California at Berkeley, 1990.

[30] N. Minorski, Nonlinear Oscillations, Van Nostrand, New York, 1962.

[31] D.D. Novaes And H. Ponce, A simple solution to the Braga-Mello conjecture, arXiv:1405.4435, to appear in Int. J. Bifurc. Chaos, 2015.

[32] A. Tonnelier, The McKean's caricature of the FitzHugh-Nagumo model I. The spaceclamped system, SIAM J. Appl. Math. 63 (2003), 459-484.

[33] A. Tonnelier And W. Gerstner, Piecewise linear differential equations and integrate-and-fire neurons: Insights from two-dimensional membrane models, Phys Rev. E 67 (2003), 021908.

${ }^{1}$ Departament de Matematiques, Universitat Autònoma de Barcelona, 08193 Bellaterra, Barcelona, Catalonia, Spain

E-mail address: jllibre@mat.uab.cat, ddnovaes@mat.uab.cat

2 Departamento de Matemática, Universidade Estadual de Campinas, Rua Sérgio Baruque de Holanda, 651, Cidade Universitária Zeferino Vaz, 13083859, Campinas, SP, Brazil

E-mail address: ddnovaes@ime.unicamp.br, teixeira@ime.unicamp.br 